\title{
Design and Experimental Validation of a Hybrid Micro Tele-Manipulation System
}

\author{
Kostas Vlachos and Evangelos Papadopoulos \\ Department of Mechanical Engineering, National Technical University of Athens, \\ Heroon Polytechniou 9, 15780 Athens, Greece \\ \{kostaswl, egpapado\} @central.ntua.gr
}

\begin{abstract}
This paper presents analytical and experimental results on a new hybrid tele-manipulation environment for micro-robot control under non-holonomic constraints. This environment is comprised of a haptic tele-manipulation subsystem (macro-scale motion), and a visual servoing subsystem, (micro-scale motion) under the microscope. The first subsystem includes a 5-dof (degrees of freedom) force feedback mechanism, acting as the master, and a 2-dof micro-robot, acting as the slave. In the second subsystem, a motion controller based on visual feedback drives the micro-robot. The fact that the slave micro-robot is driven by two centrifugal force vibration micro-motors makes the presented tele-manipulation environment exceptional and challenging. The unique characteristics and challenges that arise during the micromanipulation of the specific device are described and analyzed. The developed solutions are presented and discussed. Experiments show that, regardless of the disparity between master and slave, the proposed environment facilitates functional and simple micro-robot control during micromanipulation operations.
\end{abstract}

Keywords: micro-robotic mechanism, haptic mechanism, tele-manipulation, non-holonomic constraints.

\section{Introduction}

Recently, research in the area of robotic manipulation in the micro- and nano-worlds has gained a lot of interest and importance. The research activity focuses in areas such as microsurgery, direct medical procedures on cells, biomechatronics, micromanufacturing, and micro-assembly, where tele-operated micro-robotic devices can be used. It is well known now that not only the visual but also the haptic feedback can be helpful for a successful tele-operated micromanipulation procedure, [1]. Therefore, some of the master manipulators are haptic devices, able to drive the micro-robots and at the same time to transmit torques and forces to the operator.

A haptic tele-operation system, for use in microsurgery, was presented by Salcudean and Yan, [2], and by Salcudean, et al., [3]. Their system consists of two magnetically levitated and kinematically identical wrists, acting as a macro-master and a microslave, and a conventional manipulator that transports them. A tele-nano-robotics system using an Atomic Force Microscope (AFM), as the nano-robot, has been proposed 
by Sitti and Hashimoto, [4]. The system provides a 1-dof force feedback device for haptic sensing, using a linear scaling approach. A microsurgical tele-robot is presented, which consists of 6-dof parallel micromanipulator attached to a macro-motion industrial robot, and a 6-dof haptic master device, [5]. The system provides a disturbance observer to enhance the operator's perception.

A micro tele-operation system for tasks, such as micro-assembly or micromanufacturing, was developed by Ando et al., [6]. The haptic master is a 6-dof serial link mechanism, and the slave is a parallel mechanism. Alternatively the Phantom, a commercial haptic interface, can be used as a master device, [7]. The Phantom was used as a haptic master by Menciassi et al., where a micro-instrument for microsurgery or minimally invasive surgery was tested, [8]. Sitti et al. used the same haptic interface to tele-operate a piezoresistive AFM probe used as a slave manipulator and force sensor, [9]. A bio-micromanipulation system for biological objects such as embryos, cells or oocytes was presented in [10]. The system uses a Phantom to provide an augmented virtual haptic feedback during cell injection. A similar system for microinjection of embryonic stem cells into blastocysts is described in [11], although the system has no haptic feedback. The mechanical design of a haptic device integrated into a mobile nano-handling station is presented in [12]. The Delta haptic device was proposed as a nano-manipulator in [13]. This device is also interfaced to an AFM.

The proposed tele-manipulation environment is designed by taking into account a micro-manipulation scenario. According to this, a micro-manipulation task consists of two phases. In the first phase, the micro-robot executes a macro-scale motion towards a target. In the second phase, the platform executes micro-scale motions, and the micro-assembly or micro-manipulation task is performed in the field-of-view of a microscope. While the first phase demands increased velocity, the second phase requires increased motion resolution. Consequently, the proposed environment consists of two subsystems. The first subsystem, which is first introduced in [14], is a haptic telemanipulation master-slave system, responsible for the macro-scale motion of the micro-robot. The commanding master device is a 5-dof force feedback mechanism, while the executing slave is a non-holonomic 2-dof micro-robot with special behavior and driven by two centrifugal force actuators. This slave mechanism has a number of advantages relative to other micro-robotic devices; namely, it is characterized by low cost, complexity and power consumption. A detailed analysis of the micro-robot can be found in [15]. In this paper, the $3^{\text {rd }}$ generation of the micro-robotic device is presented, and a brief description of the kinematics and dynamics are given. A novel methodology that compensates for the non-holonomic constraints is also presented. The second subsystem is responsible for the micro-scale motion of the micro-robot in the field-of-view of a microscope. It includes a video-microscope that records the motion of the micro-robot, the acquired images are transmitted to the visual servoing controller, and the calculated control values are transmitted wirelessly to the microrobot. The use of the proposed hybrid tele-manipulation environment is illustrated by several experiments. These show that, regardless of the disparity between master and slave, the proposed environment facilitates functional and simple to the user microrobot control during micromanipulation operations. 


\section{Haptic Subsystem of the Micro Tele-Manipulation Environment}

The first subsystem of the tele-manipulation environment employs an existing 5-dof haptic mechanism as the master and a 2-dof micro-robotic platform driven by two centrifugal force actuators as the slave. A brief description of the master is given next.

\subsection{Haptic Master Device}

The master device is the haptic mechanism shown in Fig. 1. It consists of a 2-dof, 5bar linkage and a 3-dof spherical joint. All dof are active. To reduce mechanism moving mass and inertia, all actuators are mounted on the base. The transmission system is implemented using tendon drives with capstans. Although this haptic device was not developed for micromanipulation, it is suitable for it, since it has been designed optimally to exhibit maximum transparency, as seen from the operator side, [16]. The mechanism handle can translate in the $\mathrm{X}$ - and $\mathrm{Y}$-axes by $10 \mathrm{~cm}$, rotate about the $\mathrm{X}$ axis by $\pm 180^{\circ}$, and about the $\mathrm{Y}$ and $\mathrm{Z}$ axis by $\pm 30^{\circ}$, maintaining an excellent functionality.

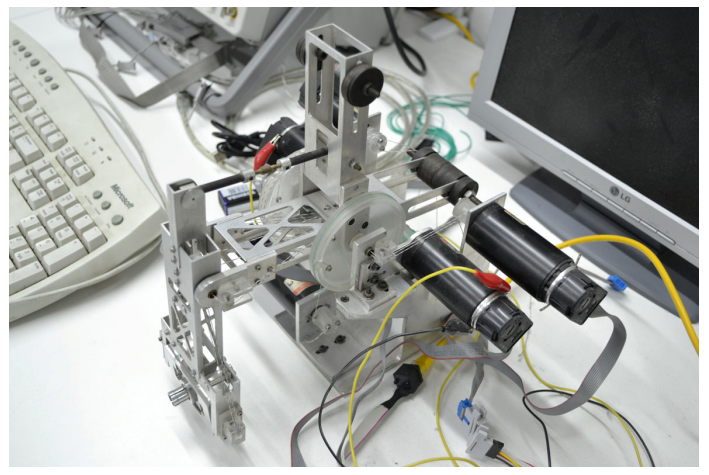

Fig. 1. The haptic master device

Employing a Lagrangian formulation yields the following mechanism equations of motion:

$$
\mathbf{M}(\mathbf{q}) \ddot{\mathbf{q}}+\mathbf{V}(\mathbf{q}, \dot{\mathbf{q}})+\mathbf{G}(\mathbf{q})=\tau+\mathbf{J}^{T} \mathbf{F}_{T}
$$

where $\mathbf{q}$ is a vector containing the five joint angles, $\mathbf{J}$, and $\mathbf{M}$ are the mechanism $5 \times 5$ Jacobian, and mass matrix respectively, $\mathbf{V}$ contains the nonlinear velocity terms, and $\mathbf{G}$ is the gravity torques vector. The vector $\boldsymbol{\tau}$ contains joint input torques while the vector $\mathbf{J}^{T} \mathbf{F}_{T}$ resolves, to the five joints, the forces and torques applied to the mechanism endpoint. The device is thoroughly described, including kinematics and dynamics, in [17]. 


\subsection{Micro Slave Device}

The 3rd generation of a new mobile micro-robot originally introduced in [15], is employed in this paper. Therefore, a more detailed description is given next.

\section{Motion Principle}

A simplified 1-dof mobile platform of mass $\mathrm{M}$ is used, whose motion mechanism employs an eccentric mass $m$, rotated by a platform mounted motor $O$, as shown in Fig. 2. One cycle of operation is completed when the mass $m$ has described an angle of $360^{\circ}$.

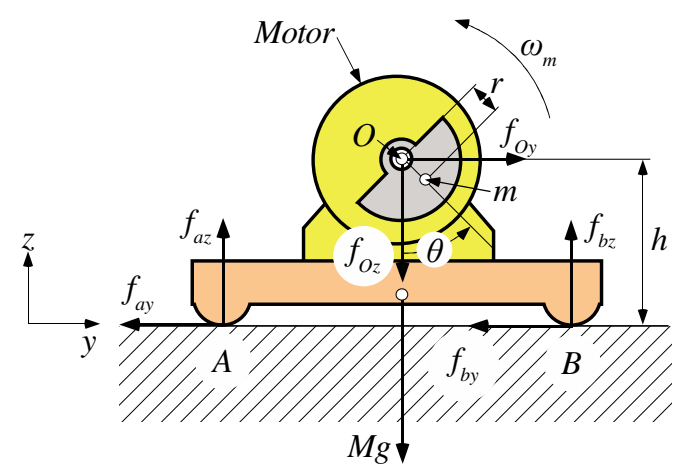

Fig. 2. Simplified 1-dof platform with rotating mass $m$

Gravitational and centrifugal forces exerted on the rotating mass are resolved along the $y-$, and z-axes to yield:

$$
\begin{aligned}
& f_{O y}=m r \omega_{m}^{2} \sin \theta \\
& f_{O z}=-m g-m r \omega_{m}^{2} \cos \theta
\end{aligned}
$$

where $\omega_{m}$ is the actuation speed, $\theta$ is the rotation angle of the eccentric mass, $g$ is the acceleration of gravity and $r$ the length of the link between $m$ and $O$. Above a critical value of actuation speed, $\omega_{\text {critical }}$, the actuation (centrifugal) forces overcome frictional forces and motion is induced. For counterclockwise rotation of the eccentric mass $m$, the platform exhibits a net displacement towards the positive y-axis. It has been shown analytically that the motion step the platform exhibits over a cycle of operation can be made arbitrarily small depending on the actuation speed $\omega$, [15]. In practice, open-loop motion resolution is limited by the electronic driving modules and by the unknown non-uniform distribution of the coefficient of friction $\mu$ along the surface of the planar motion.

\section{Platform Dynamics}

The actuation principle mentioned above was employed to the design and implementation of a 2-dof micro-robot as shown in Fig. 3 (left). 
The platform dynamics are presented in a compact matrix form by the Newton Euler equations:

$$
\begin{gathered}
M \dot{\mathbf{v}}=\mathbf{R} \sum_{i}{ }^{b} \mathbf{f}_{i}, \quad i=\{A, B, C, D, E\} \\
I_{z z} \ddot{\psi}=\hat{\mathbf{z}} \sum_{i}\left({ }^{b} \mathbf{r}_{i} \times{ }^{b} \mathbf{f}_{i}\right), \quad i=\{A, B, C, D, E\}
\end{gathered}
$$

where $b$ is the body-fixed frame, $\mathbf{R}$ is the rotation matrix between frame $b$ and the inertial frame $O, \ddot{\psi}$ is the platform angular velocity, and $\mathbf{v}=[\dot{x}, \dot{y}, \dot{z}]^{T}$ is its center of mass $(\mathrm{CM})$ velocity with respect to the inertial frame $O$. In (4), $I_{z z}$ is the polar moment of inertia in the body fixed frame and $\hat{\mathbf{z}}$ denotes the unit $\mathrm{z}$-axis vector. In both equations the subscripts $i=\{A, B, C\}$ correspond to frictional forces at the contact points of the platform, and $i=\{D, E\}$ correspond to the forces generated by the two vibrating motors. The actuation forces that act on the platform, when the DC micro-motors rotate (assuming identical micro-motors), are given by:

$$
\begin{aligned}
& { }^{b} f_{i x}=m r \ddot{\theta}_{i} \cos \theta_{i}-m r \dot{\theta}_{i}^{2} \sin \theta_{i} \\
& { }^{b} f_{i z}=-m g-m r \ddot{\theta}_{i} \sin \theta_{i}-m r \dot{\theta}_{i}^{2} \cos \theta_{i}
\end{aligned}
$$

where, $i=\{D, E\}$, and $\theta_{i}$ is the angle of micro-motor $i$.
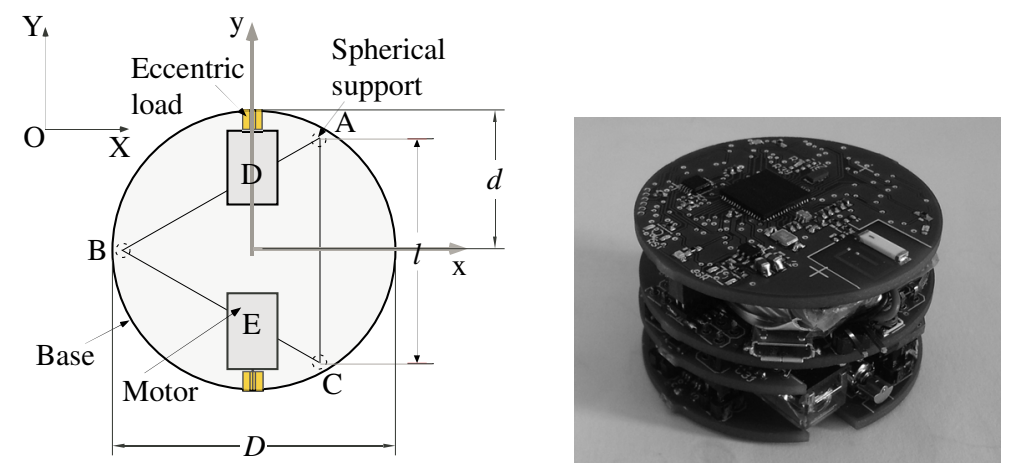

Fig. 3. Schematic design of the base (left), and the $3^{\text {rd }}$ generation micro-robot prototype (right)

\section{Micro-robot Prototype}

The $3^{\text {rd }}$ generation of the micro-robot is presented in Fig. 3 (right). This includes two vibration DC motors fed by pic-controlled H-bridges, wireless communications to a PC commanding station, a needle with force sensing capabilities, and an on-board battery. It includes advanced features, such as optical flow displacement sensors, motor speed optical sensors, and battery recharging through a USB port.

\section{Micro-robot Motion Capabilities}

Simulation runs, and experiments on the basic motion capabilities of the micro-robot, indicated that the micro-robotic platform is capable of moving forward and backward, 
in a straight or curved line, while it can rotate both clockwise and anticlockwise, [18]. Moreover, due to non-holonomic constraints, it is impossible for the platform to move parallel to the Y-axis connecting the two motors. This would be a problem during a micromanipulation procedure because the motion of the platform towards the forward direction results in a small parasitic sideways deviation. More specifically, because of unmodeled dynamics, the platform deviates towards the sidewise direction from its straightforward motion by a small amount $\Delta y$. Since the platform is incapable of moving in the sidewise direction so as to correct this parasitic effect, a method of performing such a positioning correction is developed.

It is based on the execution of a V-shaped motion, divided into two symmetrical stages. The first part of the motion is achieved when the left motor rotates in the positive direction. In the second half of the motion only the right motor rotates with positive angular velocity, see Fig. 4 (left). This specific sequence of motions results in a net displacement towards the right of the platform, see Fig. 4 (right). Reversing the two angular velocities a displacement towards the left can be achieved.
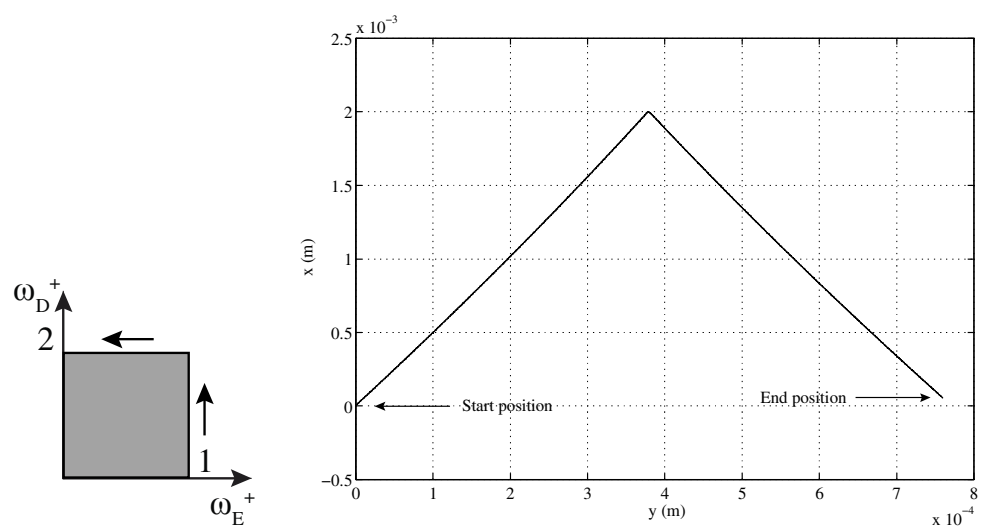

Fig. 4. Angular velocities graph for the sidewise displacement (left), and simulated motion of the platform (right)

\subsection{Haptic Tele-Manipulation Environment Analysis}

\section{Slave Micro-robot Features}

The design and special features of the slave micro-robot introduce a number of challenges that need to be tackled by the tele-manipulation environment design.

- The micro-robot is able for coarse and fine motion. Its translational sliding velocity is up to $1.5 \mathrm{~mm} / \mathrm{s}$.

- The slave micro-platform and the master haptic device are kinematical dissimilar.

- The inverse kinematics of the nonlinear micro-robot is not available in real time.

- The micro-robot exhibits complex nonholonomic characteristics.

- The vibration actuators must operate within a specific speed range (rpm). When this upper limit is exceeded, the micro-robot exhibits an additional undesirable vertical vibration. A low rpm limit also exists and is due to the need to overcome the support frictional forces, so that net motion may result. 


\section{Master Haptic Device Requirements}

The above slave micro-robot features dictate the following requirements for the master haptic device.

- The master haptic device has to drive the micro-platform towards the target in coarse motion.

- To resolve the kinematical dissimilarity between the master and the slave, a mapping from the master haptic device Cartesian space to the micro-robot actuator space has to be developed.

\section{Implementation}

In the haptic tele-manipulation environment, the master haptic device transmits motion commands to the micro-robot. PWM circuits drive the micro-platform actuators, according to the percentage (0-100\%) of their duty cycle. As a result, actuator angular velocities are set, and produce micro-robot translations and rotations. Consequently the output of the master haptic device should be the percentage $(0-100 \%)$ of the PWM duty cycle. The input to the haptic mechanism is the command given by the operator's hand. Two mutually exclusive input modes are defined. The first is the Macroscopic Input Mode (MaIM), and the second is the Macroscopic Rotation Input Mode (MRIM). The operator can choose and control the modes using the appropriate software.

\section{Macroscopic Input Mode}

The master haptic manipulator uses this mode in order to drive the micro-robotic platform towards the micro-target in linear or curved coarse motion. In this mode the positive/ negative translation of the master haptic device end-effector in the $\mathrm{X}$ axis results in increase of the positive/negative rotational speed of both micro-robot vibration micro-actuators, and therefore results in micro-robot translation along the $\mathrm{X}$ axis.

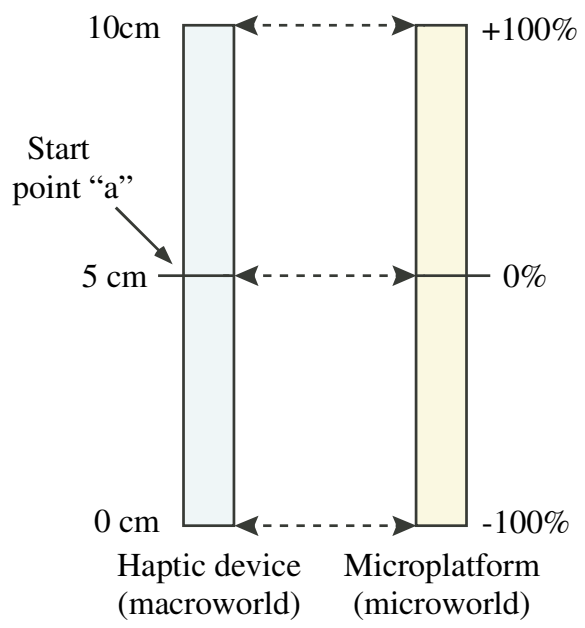

Fig. 5. The MaIM input scheme 
To obtain a curved translation, a difference in the micro-actuator rotational velocities must exist. This is achieved by rotating the haptic device end-effector about the Y axis. A positive/ negative rotation about this axis results in an increase of the rotational speed of the first/ second micro-actuator. As mentioned earlier, the haptic device end-effector can translate in the $\mathrm{X}$ axis by $10 \mathrm{~cm}$ and rotate about the $\mathrm{Y}$ axis by about $\pm 30^{\circ}$. Therefore, the start point of the end-effector is taken in the middle of its possible displacement, see Fig 5, point "a". A translation of the haptic device end-effector from start point "a" results in a percentage command of the micro-actuator speeds $q$ according to,

$$
q=20(p-5)[\%]
$$

where $p[\mathrm{~cm}]$ is the haptic device end-effector position. Additionally, for each degree $\left({ }^{\circ}\right)$ of end-effector rotation about the $\mathrm{Y}$ axis, the corresponding micro-actuator speed is increased by $1 \%$.

\section{Macroscopic Rotation Input Method}

The master haptic device uses this mode to rotate the micro-robot without translation, again in coarse motion. This mode is useful in changing fast the direction of microplatform motion, and can be achieved by rotating the micro-actuators in equal and opposite speeds. To this end, the master operator translates the end-effector along the $\mathrm{X}$ axis resulting in an increase of the rotational speed of both micro-actuators, but this time with opposite speed direction.

Table 1 illustrates the presented input modes above. The "+"/ "-" symbols denote a positive/negative rotational micro-actuator speed, the " $\uparrow$ " symbol denotes a microactuator speed increase, while " 0 " denotes that the corresponding micro-actuator is not influenced. During the MaIM phase, "1" denotes that the corresponding microactuator is functioning, " 0 " denotes that the micro-actuator is not functioning.

Table 1. Haptic tele-manipulation environment input modes

\begin{tabular}{l|cc|cc}
\hline & \multicolumn{2}{|c|}{ MaIM } & \multicolumn{2}{c}{ MRIM } \\
\hline In X positive & + & + & + & - \\
In X negative & - & - & - & + \\
About Y positive & $\uparrow$ & 0 & $\uparrow$ & 0 \\
About Y negative & 0 & $\uparrow$ & 0 & $\uparrow$ Motor B \\
\hline \multicolumn{2}{l}{} \\
\hline
\end{tabular}

As discussed earlier, above a critical micro-actuator speed, the micro-robot vibrates vertically and may even tip over. To indicate the limits of the permissible actuation speed, a spring force proportional to haptic end-effector translation (and microactuator speed) is applied to the operator. This force is given by,

$$
f_{s p}=k(p-5)
$$

where $p$ is the haptic device end-effector translation, and $k$ is a variable spring constant. It was found by experimentation that tipping occurs at about $85 \%$ of the 
maximum micro-actuator speed, depending on ground type or platform mass. To signal this limit, a spring constant three times harder than before is employed above the $85 \%$ of the maximum speed. To achieve a smooth transition, the spring constant is changing according to an exponential function. The maximum force applied to the operator is set at $5 \mathrm{~N}$. This value is slightly under the $15 \%$ of $35.5 \mathrm{~N}$, which is the average maximum controllable force a female can produce with her wrist according to Tan et al. in [19]. Measurements in [20] showed that humans exert forces up to $15 \%$ of their maximum ability, without fatigue for a long period of time. Consequently, the chosen spring constant, $k$, is defined as:

$$
k=\left\{\begin{array}{cc}
0.33 & |p-5| \leq 4.25 \\
e^{0.68(|p-5|-4.25)}-0.66 & 5.0 \geq|p-5|>4.25 \\
1 & |p-5|>5.0
\end{array}\right.
$$

\section{Visual Servoing Subsystem of the Micro Tele-Manipulation Environment}

As mentioned earlier, the proposed tele-manipulation environment is designed by taking into account a micro-manipulation scenario, where a micro-manipulation task consists of two phases. In the first phase, the micro-robot executes a macroscale motion towards a target. In the second phase, the platform executes microscale motions, and a micro-manipulation task is performed in the field-of-view of a microscope. Assuming that the micro-robot, using the first subsystem described above, has positioned in the field-of-view of the microscope, the tele-manipulation environment switches to the second subsystem. In this subsystem, the motion of the micro-robot is not controlled from the haptic device anymore. A visual servoing controller undertakes this task, described by the following set of rules:

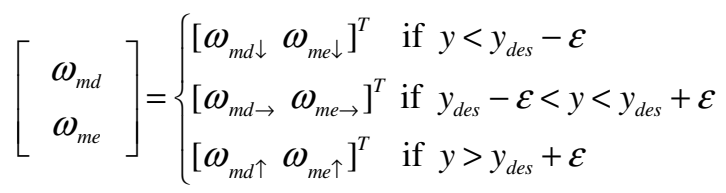

where $\left[\omega_{m d \downarrow}, \omega_{m e \downarrow}\right]^{T}$ and $\left[\omega_{m d \uparrow}, \omega_{m e} \uparrow\right]^{T}$ denote motor angular velocity pairs that result in a platform displacement with a positive or negative instantaneous curvature respectively. The vector $\left[\omega_{m d \rightarrow}, \omega_{m e \rightarrow}\right]^{T}$ denotes the pair of motor angular velocities that result in straight line translation, and $2 \varepsilon$ designates the width of the acceptable path. The specific angular velocity pair values depend on system parameters and distance from the target, and are identified by experiments. The goal for the end-effector of the micro-robot is to follow a predefined horizontal corridor-like path of width $2 \varepsilon$, reach a desired target point, and then stop. The end-effector motion is recorded by a video-microscope, and the images are transmitted to the controller. The outcome of the image processing of each frame is the plane position of the end-effector. This information is fed back to the controller, and the control inputs are calculated, according to (9). The inputs, expressed as PWM commands, are transmitted wirelessly to the microrobot and the appropriate voltages are applied to its motors. 
A graphical representation of the controller action is illustrated in Fig. 6. The colored strip represents part of the desired path. The platform is forced to translate inside the desired path strip. When the end-effector of the micro-robot reaches the target location, both motors are stopped.

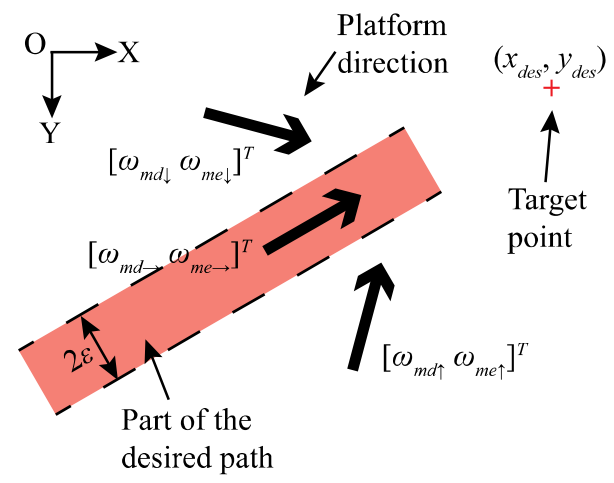

Fig. 6. Graphical representation of the controller action

\section{Simulation of the Micro Tele-Manipulation Environment}

Next, a model of the first subsystem of the micro tele-manipulation environment is defined. It consists of (a) the operator's hand, (b) the haptic mechanism, and (c) the micro-robotic system, see Fig. 7 (left). The operator's hand is modeled as a massspring-damper system, attached to the haptic mechanism modeled as a mass-damper system, see Fig. 7 (right). Note, that in the first subsystem, the haptic device is connected with a virtual spring defined by (8), with spring constant $k$.

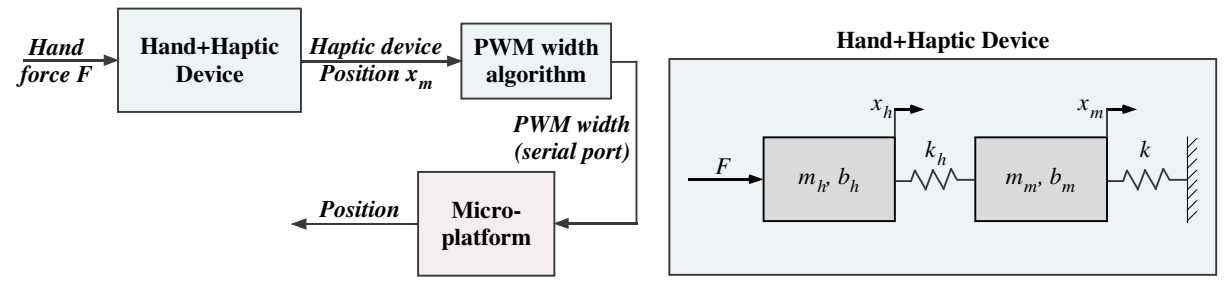

Fig. 7. The model of the haptic tele-manipulation system (left), including the user hand (right)

The transfer function of the "Hand+Haptic Device" block in Fig. 9 is described by (10), and the related symbols are defined in Table 2.

$$
\frac{X_{m}(s)}{F(s)}=\frac{k_{h}}{m_{h} m_{m} s^{4}+A s^{3}+B s^{2}+C s+k_{h} k}
$$

where $A=m_{h} b_{m}+b_{h} m_{m}, B=m_{h}\left(k+k_{h}\right)+b_{h} b_{m}+k_{h} m_{m}$, and $C=b_{h}\left(k+k_{h}\right)+k_{h} b_{m}$. 
Table 2. Definition of the symbols in (10) and Fig. 9

\begin{tabular}{cccc}
\hline Symbol & Definition & Symbol & Definition \\
\hline$F$ & Operator's hand force & $m_{m}$ & Haptic mechanism mass \\
$m_{h}$ & Operator's hand mass & $b_{m}$ & Haptic mechanism damping \\
$b_{h}$ & Operator's hand damping & $x_{m}$ & Haptic mechanism position \\
$x_{h}$ & Operator's hand position & $k$ & Virtual spring constant \\
$k_{h}$ & Operator's hand stiffness & & \\
\hline
\end{tabular}

During the simulation, the operator's hand mass $m_{h}$ is $1.46 \mathrm{Kg}$, the hand damping $b_{h}$ is $3.6 \mathrm{Ns} / \mathrm{m}$. and the hand stiffness $k_{h}$ is $200 \mathrm{~N} / \mathrm{m}$. These represent average values taken from the relevant literature, [21-22]. The haptic mechanism apparent mass $m_{m}$ in $\mathrm{X}$ axis is about $0.27 \mathrm{Kg}$, and the mechanism damping $b_{m}$ is about $5 \mathrm{Ns} / \mathrm{m}$. These values were found through experimentation with the haptic mechanism, see [17]. The input to the system is a step of about $0.18 \mathrm{~N}$ of the operator's hand force $F$. The virtual spring value $k$ is $4 \mathrm{~N} / \mathrm{m}$. The simulation run realizes the following scenario. The motion of the micro-robot starts at point $(0 \mathrm{~m}, 0 \mathrm{~m})$ employing the haptic subsystem of the micro tele-manipulation environment. Here, the haptic device drives the microrobot towards the field-of-view of the video-microscope. We assume that it begins at $(0.0001 \mathrm{~m}, 0 \mathrm{~m})$. When the micro-robot enters the field-of-view, the visual servoing subsystem takes control, and drives the micro-robot towards the desired target point at $(0.001,0)$, according to (9). As shown in Fig. 8, the proposed micro tele-manipulation environment successfully drives the micro-robot to the target point, and then stops.
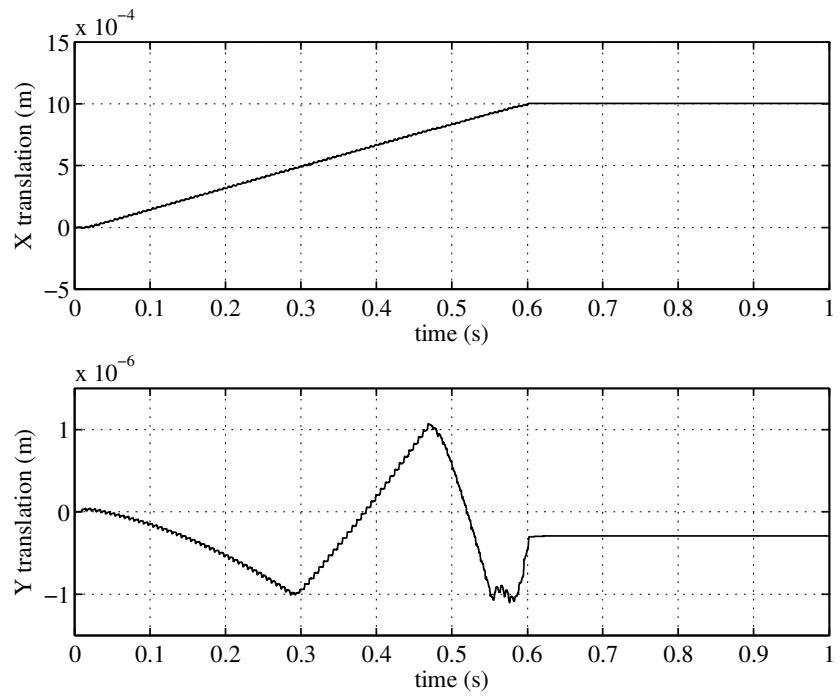

Fig. 8. The application of both subsystems of the micro tele-manipulation subsystem 


\section{Experimental Results}

To validate the proposed environment, various experiments are conducted. The goal is to use the haptic device to drive the micro-robot towards the field-of-view of the video-microscope, and then let the visual servoing controller to drive the micro-robot to a target point using a predefined path. During the first phase of the experiment the operator moves the haptic device end-effector along the $\mathrm{X}$ axis and rotates it about the $\mathrm{Y}$ axis. The end-effector position and the angle are captured by encoders attached on the haptic device actuators (Maxon dc motors), and transmitted to a PC/104 tower. This tower is the control unit, running the algorithm that translates the operator input into the micro-robot input according to (6). At the same time the haptic device applies forces to the operator according to (7) and (8).

When the micro-robot reaches the field-of-view of the video-microscope, the second phase of the experiment begins, which is realized by the second subsystem of the micro tele-manipulation environment. The motion of the end-effector of the micro-robot is recorded by a video-microscope. The video camera pixel size was chosen so that the measurement resolution of the system is approximately $2 \mu \mathrm{m}$. The video camera selected was the Marlin F146B, from Allied Vision Technologies, GMBH. The acquired images are transmitted via a FireWire 400 port to a Core 2, $2 \mathrm{GHz}$ PC laptop, and processed on-the-fly in Matlab. The outcome of the image processing of each frame is the plane position of the end-effector of the micro-robot. This information is fed back to the controller, and the control inputs are calculated, according to (9). The inputs, expressed as PWM commands, are transmitted wirelessly to the microrobot and the appropriate voltages are applied to its motors. The control loop duration is $80 \mathrm{~ms}$. The path of the end-effector of the micro-robot under the microscope is shown in Fig. 9a. The desired target point is marked with the red "plus" symbol. We can see that when the end-effector enters the field-of-view of the microscope, the visual servoing controller force it to follow the predefined path towards the target.
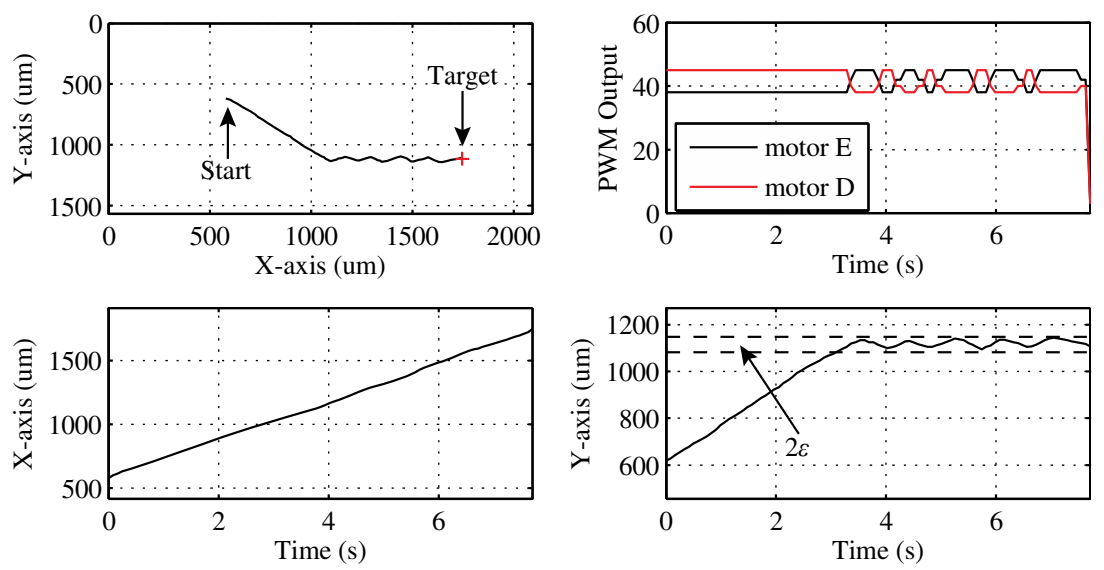

Fig. 9. (a) Path of the micro-robot end-effector, (b) PWM output from the controller, (c) $x$ trajectory of the end-effector tip, (d) y trajectory of the end-effector tip 


\section{Conclusions}

The analysis and several experimental results of a new hybrid micro-robot telemanipulation environment are presented in this paper. The proposed environment combines two different subsystems. The first subsystem, employed during the coarse motion of the micro-robot, includes a 5-dof force feedback mechanism, acting as the master, and a 2-dof micro-robot, acting as the slave. Regardless of the disparity between master and slave and the fact that the slave micro-robot is driven by two centrifugal force vibration micro-motors, the environment gives to the operator the ability to drive and control the micro-platform in a functional and simple manner. In the second subsystem, a motion controller based on visual feedback drives the microrobot in a predefined path under the field-of-view of a video-microscope.

The proposed environment manages to solve with success problems that arise during micromanipulation with the specific micro-robot, such as that the slave microplatform and the master haptic device are kinematical dissimilar, that the vibration actuators must operate within a specific speed range (rpm), and that they must achieve high speed during the macroscopic motion and sub-micrometer positioning accuracy during the microscopic motion.

\section{References}

1. Salcudean, S.E., Ku, S., Bell, G.: Performance measurement in scaled teleoperation for microsurgery. In: Troccaz, J., Mösges, R., Grimson, E. (eds.) CVRMed-MRCAS 1997. LNCS, vol. 1205, pp. 789-798. Springer, Heidelberg (1997)

2. Salcudean, S.E., Yan, J.: Towards a Force-Reflecting Motion-Scaling System for Microsurgery. In: Proc. IEEE Int. Conf. on Robotics and Automation (ICRA 1994), San Diego, CA, USA, pp. 2296-2301 (1994)

3. Salcudean, S.E., Wong, N.M., Hollis, R.L.: Design and Control of a Force-Reflecting Teleoperation System with Magnetically Levitated Master and Wrist. IEEE Trans. on Robotics and Automation 11(6), 844-858 (1995)

4. Sitti, M., Hashimoto, H.: Tele-Nanorobotics Using Atomic Force Microscope. In: Proc. IEEE/RSJ Int. Conf. on Intelligent Robots and Systems, Victoria, B.C., Canada, pp. 1739-1746 (1998)

5. Kwon, D.S., Woo, K.Y., Cho, H.S.: Haptic Control of the Master Hand Controller for a Microsurgical Telerobot System. In: Proc. IEEE Int. Conf. on Robotics and Automation (ICRA 1999), Detroit, Michigan, USA, pp. 1722-1727 (1999)

6. Ando, N., Ohta, M., Hashimoto, H.: Micro Teleoperation with Haptic Interface. In: Proc. of 2000 IEEE Int. Conf. on Industrial Electronics, Control and Instrumentation (IECON 2000), Nagoya, Japan, pp. 13-18 (2000)

7. Massie, T., Salisbury, J.K.: The Phantom Haptic Interface: A Device for Probing Virtual Objects. In: Proc. ASME Winter Annual Meeting, Symposium on Haptic Interfaces for Virtual Environment and Teleoperator Systems, Chicago, IL, pp. 295-301 (1994)

8. Menciassi, A., Eisinberg, A., Carrozza, M.C., Dario, P.: Force Sensing Microinstrument for Measuring Tissue Properties and Pulse in Microsurgery. IEEE/ASME Trans. on Mechatronics 8(1), 10-17 (2003) 
9. Sitti, M., Aruk, B., Shintani, H., Hashimoto, H.: Scaled Teleoperation System for Nano-Scale Interaction and Manipulation. Advanced Robotics 17(3), 275-291 (2003)

10. Ammi, M., Ferreira, A.: Realistic Visual and Haptic Rendering for Biological-Cell Injection. In: Proc. IEEE Int. Conf. on Robotics and Automation (ICRA 2005), Barcelona, Spain, pp. 930-935 (2005)

11. Mattos, L., Grant, E., Thresher, R.: Semi-Automated Blastocyst Microinjection. In: Proc. IEEE Int. Conf. on Robotics and Automation (ICRA 2006), Orlando, Florida, USA, pp. 1780-1785 (2006)

12. Kortschack, A., Shirinov, A., Trueper, T., Fatikow, S.: Development of Mobile Versatile Nanohandling Micro-robots: Design, Driving Principles, Haptic Control. Robotica 23(4), 419-434 (2005)

13. Grange, S., Conti, F., Helmer, P., Rouiller, P., Baur, C.: The Delta Haptic Device as a Nanomanipulator. Proc. SPIE Micro-Robotics \& Microassembly III 4568, 100-111 (2001)

14. Vlachos, K., Papadopoulos, E.: Analysis and Experiments of a Haptic Tele-Manipulation Environment for a Microrobot Driven by Centripetal Forces. ASME Journal of Computing Sciences and Information in Engineering - Special Issue on Haptics, Tactile and Multimodal Interfaces 8(4) (2008)

15. Vartholomeos, P., Papadopoulos, E.: Dynamics, Design and Simulation of a Novel Microrobotic Platform Employing Vibration Microactuators. Journal of Dynamic Systems, Measurement and Control, ASME 128, 122-134 (2006)

16. Vlachos, K., Papadopoulos, E.: Transparency Maximization Methodology for Haptic Devices. IEEE/ASME Trans. on Mechatronics 11(3), 249-255 (2006)

17. Vlachos, K., Papadopoulos, E., Mitropoulos, D.: Design and Implementation of a Haptic Device for Urological Operations. IEEE Trans. on Robotics \& Aut. 19(5), 801-809 (2003)

18. Vlachos, K., Vartholomeos, P., Papadopoulos, E.: A Haptic Tele- Manipulation Environment for a Vibration-Driven Micromechatronic Device. In: Proc. IEEE/ASME International Conference on Advanced Intelligent Mechatronics Systems (AIM 2007), ETH Zurich, Switzerland, September 4-7 (2007)

19. Tan, H.Z., Srinivasan, M.A., Ederman, B., Cheng, B.: Human Factors for the Design of Force-Reflecting Haptic Interfaces. ASME Dynamic Systems and Control 55(1), 353-359 (1994)

20. Wiker, S.F., Hershkowitz, E., Zilk, J.: Teleoperator Comfort and Psychometric Stability: Criteria for Limiting Master-Controller Forces of Operation and Feedback During Telemanipulation. In: Proc. NASA Conference on Space Telerobotics, Pasadena, CA, USA, vol. I, pp. 99-107 (1989)

21. Gil, J.J., Avello, A., Rubio, A., Florez, J.: Stability Analysis of a 1 DOF Haptic Interface Using the Routh-Hurwitz Criterion. IEEE Trans. on Control System Technology 12(4), 583-588 (2004)

22. Salcudean, S.E., Zhu, M., Zhu, W.-H., Hashtrudi-Zaad, K.: Transparent Bilateral Teleoperation under Position and Rate Control. I. J. Robotic Research 19(12), 1185-1202 (2000) 